\title{
MEDICIÓN DE INTELIGENCIA EN ADOLESCENTES Y ADULTOS SEGÚN LA MODALIDAD DE APLICACIÓN: TRADICIONAL E INFORMATIZADA
}

\author{
MEASUREMENT OF INTELLIGENCE IN TEENAGERS AND ADULTS BY METHOD \\ OF APPLICATION: TRADITIONAL AND COMPUTERIZED
}

AyC. CONSULTORA DE RECURSOS HUMANOS Y ASESORÍA EMPRESARIAL

Christiam Gamarra T. ${ }^{1}$

(RECIBIDO 25/06/2012, ACEPTADO 02/11/2012)

\begin{abstract}
RESUMEN
En un inicio se aplicó la prueba de inteligencia de Raven a un grupo de 240 sujetos, entre adolescentes estudiantes y adultos trabajadores de universidades (docentes y tutores de cursos), luego de esta primera evaluación se seleccionó a 120 sujetos cuyos puntajes directos sean homogéneos (media igual a 29, considerando aquellos cuyo puntaje mínimo es de 27 y máximo de 31). En este segundo grupo se aplicó la prueba de inteligencia "Toni 2", a la mitad de ellos del modo tradicional, con lápiz y papel impreso de la prueba, al resto se le aplicó la versión informatizada, diseñada en el programa Excel 2010 (para el diseño de la versión informatizada se tuvo en cuenta el hecho de no dejar a lado ningún detalle original de la prueba, cuya variación fueron las instrucciones de aplicación).

Comparando los resultados generales se encontró que el grupo de evaluación informatizada consiguió una media mayor a comparación del grupo que desarrollo la prueba en el modo tradicional, diferencia que no se manifestaba en sus resultados de la prueba inicial. Del mismo modo, se encuentra que en el grupo de adolescentes la diferencia es aún mayor, encontrándose diferencia significativa en la diferencia de sus medias con respecto a la modalidad de aplicación, en el grupo de adultos existe diferencia que no es significativa.
\end{abstract}

Otro dato interesante encontrado en la investigación es que los puntajes de validez de ítems y confiabilidad de la prueba son diferentes en ambos grupos. En términos generales, se encuentran mayores puntajes de confiabilidad en la versión informatizada, siendo la diferencia más remarcada en el grupo de adolescentes (presenta mayor índice de confiabilidad).

Palabras clave: inteligencia, medición, test informatizados, confiabilidad, validez.

1 Gerente General de AYC - Consultora de recursos humanos y asesoría empresarial. E-mail: chris_heart@hotmail.com 


\begin{abstract}
Initially we applied the Raven intelligence test to a group of 240 subjects, among adolescent's students and adults, university employees (teachers and tutors of courses), after this first evaluation we selected 120 subjects whose scores are homogeneous direct (mean equal to 29 , whereas those whose minimum score is 27 and maximum of 31 ).

In this second group intelligence test applied "Toni 2, " half of them the traditional way with pencil and paper test form, the rest was applied to the computerized version, designed in Excel 2010 (for the design of the computerized version took into account the fact of not leaving any details aside from the original test, whose change was the application instructions). Comparing the overall results found that computerized assessment group got more than half compared to the group that developed the test in the traditional way; this difference was not manifested in their initial test results. Similarly we find that the adolescent group the difference is even greater, significant differences in the difference of their means with regard to the mode of application, in the adult group there is no significant difference.

Another interesting research found is that the scores of validity and reliability of items of evidence are different in both groups.

Overall scores are higher reliability in the computerized version, with the difference highlighted in the adolescent group (a higher rate of reliability).
\end{abstract}

Keywords: Intelligence, measurement, test computer, reliability, validity.

\title{
INTRODUCCIÓN
}

Nuestra sociedad afronta un gran cambio en los diferentes hábitos y estilos de vida, la tecnología juega un papel importante en el desarrollo de nuestras vidas diarias, tecnología que ha tenido grandes avances en un tiempo corto. Como es el impacto de las redes sociales, que en un plazo no mayor a cinco años ha logrado abarcar gran parte de la vida cotidiana de las personas. Esta revolución informática ha provocado que la modalidad de evaluación, con respecto a la aplicación de test, se adapte a la tecnología actual, en donde ahora se utilizan diversos tipos y modalidades de los ya famosos "test informatizados"; no hay duda alguna que las ventajas que nos ofrecen esta modalidad de evaluación son muy amplias, en tal sentido que son cada vez más los profesionales psicólogos que prefieren la utilización de estas herramientas; sin embargo, el ajuste de estas herramientas demanda una calibración de estos instrumentos, debido a que las condiciones de evaluación varían (y muchos profesionales no toman en cuenta los aspectos o variables que pueden intervenir en el proceso de evaluación informatizada) y por ende, es necesario ajustar nuestros instrumentos, puestos en esta modalidad, para poder hacer evaluaciones más certeras y precisas que no se alejen de la realidad objetiva de los sujetos.

En el presente trabajo planteamos el hecho de la necesidad de calibrar la nueva modalidad de evaluación, en base a test informatizados, ya que en un grupo (homogenizado) se plantearon los dos tipos de evaluación (tradicional e informatizada) 
y al margen de encontrar diferencias en sus resultados generales, encontramos diferencias en los puntajes de confiabilidad y validez de los test. Por ello, que es necesario fomentar los estudios con respecto a las nuevas formas de evaluación y poder tener mejores herramientas de evaluación que ayuden al trabajo diario del profesional psicólogo.

\section{Problema}

En la actual sociedad en que vivimos, la tecnología juega un papel de suma importancia para el desarrollo personal y profesional de las personas, acorde a esa realidad, la psicología viene adaptándose a ese cambio tecnológico que sigue creciendo a grandes pasos; dentro de ese proceso se viene desarrollando con gran fuerza la aplicación de test informatizados, ya sea por medio plantillas de corrección, software psicológico o test on-line por medio de páginas web. Muchas compañías, instituciones educativas, psicólogos particulares aplican estas pruebas a los sujetos, pero el tema que está en controversia es si mediante esta modalidad se puede hablar de la misma validez y confiabilidad del instrumento, las variables que influyen en la aplicación de esta modalidad de test es muy cambiante, las indicaciones difieren del aplicativo tradicional y es de suma importancia comenzar a diferenciar la influencia de características de la aplicación informatizada (software que se utiliza, brillo de la pantalla, pulgadas de la pantalla, estado del mouse, teclado, etc.) variables que pueden influenciar sobre la aplicación y es necesario controlar y obtener las mejores condiciones para su funcionamiento adecuado, dicho estudio aún está pendiente.

Es necesario e imprescindible adecuar nuestros instrumentos de evaluación, si cambia la modalidad de aplicación es imprescindible desarrollar la estandarización correspondiente manejando las nuevas variables que intervienen en este proceso.

Con respecto a estas comparaciones, dentro de la investigación presente, podremos diferenciar los puntajes obtenidos con respecto al coeficiente intelectual en ambos tipos de aplicación, (teniendo una evaluación previa con un test diferente que mida el mismo constructo, para seleccionar un grupo homogéneo), del mismo modo se procedió a realizar el análisis de confiabilidad y validez de ítems de la prueba, comparando los tipos de aplicación en cada grupo.

\section{Problema general}

¿Existe diferencia significativa con el puntaje de inteligencia alcanzado en un grupo según la modalidad de aplicación tradicional e informatizada?

\section{Problemas específicos}

- ¿Existe diferencia significativa con respecto a la modalidad de aplicación tradicional e informatizada según la edad de los sujetos?

- ¿Existe diferencia en los puntajes de confiabilidad y validez de ítems en los grupos según la modalidad de aplicación del test? 


\section{Objetivos}

\section{Objetivo general}

Demostrar que existe diferencia significativa con el puntaje de inteligencia alcanzado en un grupo según la modalidad de aplicación tradicional e informatizada.

\section{Objetivo específicos}

- Identificar la existencia de diferencia significativa con respecto a la modalidad de aplicación tradicional e informatizada según la edad de los sujetos.

- Identificar la existencia de diferencia en los puntajes de confiabilidad y validez de ítems en los grupos según la modalidad de aplicación del test.

\section{Marco teórico}

\section{Sobre inteligencia}

Son tantas los trabajos, estudios e investigaciones sobre la inteligencia humana que se tiene abundante información sobre el desarrollo de este constructo. Por ende, seremos breves en este apartado.

\section{Antecedentes}

En un inicio, la inteligencia se consideró como algo más bien biológico, hereditario, que estaba dentro de la mente del individuo y que podía medirse; es decir, atributo unitario situado en la cabeza de los individuos. Para Thorndike (1920) es: "La habilidad para comprender y dirigir a los hombres y mujeres, muchachos y muchachas, y actuar sabiamente en las relaciones humanas".

Spearman (1927) sostenía que es más fácil medir la inteligencia que definirla. Además existen otros dos tipos de inteligencias: la abstracta, habilidad para manejar ideas, y la mecánica, habilidad para entender y manejar objetos.

Durante el auge del conductismo, se entra en una larga etapa que se deja de lado los procesos no directamente observables como la inteligencia, proceso que tuvo grandes décadas apartada a este constructo hasta que el científico Wechsler (1955) sostenía que la inteligencia era "La capacidad agregada y global del individuo para actuar con propósito, pensar racionalmente y para enfrentarse de modo efectivo con su medio ambiente". Ante esta definición es posible yuxtaponer la noción de inteligencia cognitiva al lado de la inteligencia no-cognitiva (emocional, personal y social).

El debilitamiento de las posturas conductistas y la emergencia de los procesos cognitivos aparecen con el estructuralismo. Los trabajos de Piaget y su teoría sobre el desarrollo intelectual contrastan visiblemente de las posturas psicometristas.

En el continuar evolutivo del estudio de la inteligencia, aparece el modelo computacional y el interés por el estudio de la inteligencia artificial. Con ello llega, a nuestro juicio, uno de los errores más graves de las teorías cognitivas. Cuando se estudian los procesos cognitivos sobre inteligencia artificial lo que estudiamos es la inteligencia computacional y no humana. 
La idea que subyace a la inteligencia actualmente es la capacidad de adaptación (Stemberg, 1997). Es como si la idea de la globalización hubiese llegado a este campo de estudio de la psicología, donde la inteligencia está muy vinculada con la emoción, la memoria, la creatividad, el optimismo, la personalidad y en cierto sentido con la salud mental.

\section{Definición}

Howard Gardner define la inteligencia como la capacidad de resolver problemas o elaborar productos que sean valiosos en una o más culturas.

Los estudios realizados por este autor son importantes ya que amplía el campo de lo que es la inteligencia y reconoce lo que todos sabíamos intuitivamente, y es que la brillantez académica no lo es todo. A la hora de desenvolvernos en esta vida no basta con tener un gran expediente académico. Hay gente de gran capacidad intelectual pero incapaz de, por ejemplo, elegir bien a sus amigos y, por el contrario, hay gente menos brillante en el colegio que triunfa en el mundo de los negocios o en su vida personal. Triunfar en los negocios, o en los deportes, requiere ser inteligente, pero en cada campo utilizamos un tipo de inteligencia distinto. No mejor ni peor, pero si distinto. Dicho de otro modo, Einstein no es más inteligente que Michel Jordan, pero sus inteligencias pertenecen a campos diferentes. Otro aspecto importante dentro de los estudios de Gardner, es que define la inteligencia como una capacidad. Hasta hace muy poco tiempo la inteligencia se consideraba algo innato e inamovible. Se nacía inteligente o no, y la educación no podía cambiar ese hecho. Tanto es así que en épocas muy cercanas a los deficientes psíquicos no se les educaba, porque se consideraba que era un esfuerzo inútil.

Al definir la inteligencia como una capacidad Gardner la convierte en una destreza que se puede desarrollar. Gardner no niega el componente genético.

Todos nacemos con unas potencialidades marcadas por la genética. Pero esas potencialidades se van a desarrollar de una manera o de otra dependiendo del medio ambiente, nuestras experiencias, la educación recibida, etc.

\section{Evaluación de la inteligencia}

La evaluación de la inteligencia nace en las evaluaciones de Cattell, es una característica de suma importancia para la evaluación psicológica actual. Es sabido que las escalas de Weschler son las más utilizadas en este medio, en donde arrojan coeficientes intelectuales más certeros que en algunas otros test de evaluación de inteligencia.

El proceso de evaluación de inteligencia parte desde pruebas sencillas de evaluación de inteligencia como el Raven prosiguiendo una línea de evolución de complejidad llegando a las escalas de Weschler antes mencionadas.

Existen gran cantidad de pruebas para medir inteligencia que están surgiendo a partir de las necesidades de practicidad y rapidez que hoy en día se necesita, pruebas como el Toni A, RP30, que fusionan ciertas características antiguas con modernas para plantear nuevos enfoques de evaluación de inteligencia. 
Ahora es cierto que el desarrollo del concepto de inteligencia, lo mismo que la teoría de la inteligencia, ha venido evolucionando a grandes rasgos, del mismo modo el proceso de evaluación de la misma ha tenido grandes avances en la psicología actual.

A comienzos del siglo XX, el foco de atención se centró en lograr instrumentos que midieran las diferencias de inteligencia. El primer autor que destacó en este sentido fue James Mc. Keen Cattell (1860-1944), quien desarrolló una batería de test para evaluar la inteligencia en las personas, y fue pionero en el uso del concepto de prueba mental.

Otro autor fue Alfred Binet que argumentaba que no se puede dar una sola definición de inteligencia, sino que deben sumarse las diferentes aptitudes mentales. Aunque Binet no negaba la influencia de los factores genéticos, consideraba también la influencia social. Así, pensaba, que los sujetos poco inteligentes padecían un retraso en su desarrollo (el conocido como retraso mental).

El mayor avance de esos investigadores lo supuso la creación del concepto de edad mental, es decir, la edad cronológica que corresponde a un nivel característico de rendimiento.

Asimismo, Stern formuló el concepto de cociente intelectual, que es el resultado de dividir la edad mental, entre la edad real y multiplicar el resultado por 100.

Siguiendo los pasos de Binet, Charles Spearman (1863-1945) estaba de acuerdo, en que la inteligencia no podía ser tomada como una cualidad general, sino que debía ser definida en función de dos factores primarios, de los que dependen todos los demás. De esta forma defendió una teoría bifactorial en la que existe un factor "g", la inteligencia general (capacidad de establecer, crear y aplicar relaciones entre los conocimientos adquiridos a un nivel abstracto), y factores "s", habilidades y capacidades responsables de las diferencias entre puntuaciones en diferentes tareas.

Posteriormente, Thurstone, en 1938, sostuvo también que la inteligencia no puede concebirse como un concepto unitario, restó importancia a un factor general, y valoró, sin embargo, las capacidades más específicas, añadiendo que la inteligencia estaría formada por siete factores o aptitudes mentales primarias:

Comprensión Verbal: Es la capacidad para comprender material verbal, donde los test más usados son los de vocabulario y comprensión lectora.

Fluidez Verbal: Consiste en la producción de palabras, sentencias y textos, por ejemplo, recordar palabras que comiencen por la letra A en un tiempo determinado.

Habilidad Numérica: Realizar cálculos numéricos y resolver problemas simples, con rapidez y precisión.

Memoria: Aptitud que supone en el recuerdo de series de palabras, letras, números o rostros. Se mide repitiendo palabras, números, etc. Mide la memoria a corto plazo.

Rapidez Perceptiva: Esta aptitud implica el reconocimiento rápido de letras y números y en la capacidad de señalar semejanzas y diferencias entre distintos dibujos. 
Visualización Espacial: Consiste en la visualización de formas, rotación de objetos y tareas en las que se necesita encajar las piezas de un puzzle.

Razonamiento Inductivo: Esta aptitud implica la capacidad de razonar a partir de lo particular para llegar a lo general. Se mide con test de series de letras, serie de números y clasificación de palabras. Es la capacidad de ir de lo concreto a lo general.

El concepto científico "inteligencia” se deriva de una observación sistemática del comportamiento de la gente.

El método más óptimo para comprender un concepto científico como la inteligencia consiste en comparar rigurosamente el comportamiento de distintas personas en situaciones que consideremos intelectualmente exigentes.

Las personas, como sucede con las cosas, se conocen por sus propiedades. Estas propiedades de la inteligencia se pueden descubrir a través de la observación de distintas personas actuando en distintas situaciones.

Las comparaciones entre personas pueden hacerse, a la larga, entre una persona en concreto y un determinado grupo humano.

Un test es un instrumento utilizado para medir la inteligencia y está constituido por una serie de preguntas o tareas que se administran a individuos para comprobar si poseen una capacidad o un conocimiento determinado.

Los psicólogos han creado diferentes test para medir la inteligencia y otras capacidades, por lo que hay múltiples test con el objetivo de medir el CI (cociente intelectual). Los test no son artículos de fe, son indicadores de inteligencia que debemos saber interpretar mediante el análisis factorial, que es un proceso matemático para simplificar una matriz de correlaciones entre variables en unos pocos factores compartidos por todas ellas.

No hay acuerdo en lo que se refiere a la medición de la inteligencia. Algunos psicólogos defienden la validez de los test porque son buenos predictores del aprendizaje académico pero, como es sabido, es necesario todo un proceso riguroso, dependiendo no solo de datos estadísticos, sino dependiendo de la muestra y el contexto real donde se desarrolle la prueba y su estandarización.

\section{Sobre los test informatizados}

El término test informatizados alude a la aplicación de los test en formato virtual, en donde se representa el mismo test diseñado en físico en diversos formatos computarizados, que van desde simples plantillas de corrección en un automatizado del Excel hasta sofisticados software que facilitan y ahorran el tiempo en los procesos de calificación de los mismos.

Claro está que en el progreso evolutivo de los test han desarrollado diversas técnicas y métodos para controlar diversas variables y definir de la manera más correcta la validez y confiabilidad de estas herramientas de evaluación. No obstante se 
está llevando estos test a la versión virtual sin el control debido del proceso de calibración de los instrumentos. Del mismo modo que el método clásico de evaluación (haciendo referencia a la aplicación con pruebas en físico) el proceso de evaluación virtual informatizada necesita ser fundamentada y desarrollada con los procesos de evaluación de confiabilidad y validez, para ello es necesario ahondar con la investigación de estos procesos.

Ahora la aplicación de estos test por medio de las computadoras no significa el reemplazo del trabajo del profesional psicólogo, ya que es imprescindible la participación de este para el buen funcionamiento y correcta aplicación de los test, del mismo modo que en el formato clásico existen pautas para orientar a los evaluados, se sugiere que el científico evaluador diseñe diversas pautas de acorde al proceso que está realizando con las variabilidades correspondientes del caso. Para ello es imprescindible estandarizar nuestras herramientas evaluativas de acorde a la modalidad de aplicación de la misma.

Otro factor que influye, dentro de la aplicación informatizada, es la edad de los sujetos, ya que las "nuevas" generaciones están mucho más familiarizadas con estas tecnologías, y en verdad para ellos no existe diferencia alguna entre la aplicación tradicional y virtual, inclusive la tecnología se está haciendo cada vez mas accesible a las personas (incluyendo nuevas tecnologías como el avance en tecnología celular, tablet, etc.) que estas, en un momento determinado, prefieran la aplicación virtual a la tradicional.

Una de las grandes ventajas de los test informatizados es que ahorran gran cantidad de tiempo, facilitan el proceso de obtención de resultados. Otra ventaja de estos procesos es el ahorro de materiales impresos y la precisión en obtención de los puntajes.

\section{MÉTODO}

\section{Diseño}

El diseño de investigación en una primera parte es exploratorio, ya que se orienta a obtener información del coeficiente intelectual de un grupo de sujetos, a base de esos resultados se selecciona un grupo homogéneo (con respecto a sus puntajes y por edades), para luego pasar a un diseño descriptivo comparativo porque describe los puntajes de coeficiente intelectual obtenidos por cada grupo según la modalidad de aplicación, ya sea el método tradicional o la aplicación informatizada, además se comparan resultados entre grupos de edad, género y valores de validez y confiabilidad de los ítems.

\section{Participantes}

En un principio la muestra estuvo constituida por 240 personas, a quienes se les aplico el primer test de inteligencia, 120 adolescentes cuyas edades se encontraban entre los 15 y 17 años pertenecientes a centros preuniversitarios de Lima Metropolitana, 120 adultos cuyas edades estaban entre los 35 y 40 años, trabajadores 
de la Universidad Nacional Mayor de San Marcos y la Universidad Nacional de Ingeniería. De dicho grupo se seleccionó a 120 sujetos, quienes habían obtenido puntajes similares, con ellos se trabajó la segunda instancia de la investigación, aplicando a la mitad de ellos el test informatizado y el resto con la forma tradicional.

\begin{tabular}{lcc}
\hline \multicolumn{1}{c}{ GRUPOS DE EDADES } & PRIMERA FASE & SEGUNDA FASE \\
\hline DE 15 A 17 AÑOS & $120(50 \%)$ & $60(50 \%)$ \\
DE 35 A 40 AÑOS & $120(50 \%)$ & $60(50 \%)$ \\
\hline TOTAL & $240(100 \%)$ & $120(100 \%)$ \\
\hline
\end{tabular}

\section{Instrumentos de recolección de datos}

Para el desarrollo de esta investigación se utilizaron los siguientes instrumentos:

- Test de matrices progresivas RAVEN (Escala General)

- Test de inteligencia TONI 2 - Forma A.

\section{Procedimiento}

En un inicio se aplicó a 240 sujetos el test de matrices progresivas de J. C. Raven (escala general), dentro de dicha evaluación se seleccionaron a 120 sujetos (la mitad adolescentes y la otra de adultos) cuyos puntajes brutos eran homogéneos. Dentro de este grupo de evaluados se consideró a aquellos que el puntaje bruto mínimo es de 27 y máximo de 31 (en base a la media lograda que fue igual a 29).

Dentro de los 120 sujetos seleccionados tras la primera evaluación, el 50\% de ellos son adolescentes y el resto adultos, cada subgrupo conformado por 60 sujetos. Se aplicó a 30 sujetos de cada sub-grupo la prueba en versión informatizada (mediante plantillas automatizadas en Excel 2010, todos los sujetos rindieron su prueba en una laptop HP-Compaq, pantalla de 14 pulgadas, no fue necesario la utilización de ningún tipo de mouse, solo se utilizó el teclado, la configuración del sistema operativo fue la misma para todos los evaluados) y al resto se le aplicó la prueba de forma tradicional (con la prueba en físico).

Luego se realizó el análisis estadístico de los resultados para determinar los niveles de validez, confiabilidad y poder diferenciar entre los puntajes globales si existe $o$ no diferencia entre los grupos participantes. Para tales efectos se utilizaron programas de análisis de resultados como el Microsoft Excel 2010 y el SPSS versión 19.

\section{RESULTADOS}

Resultados de la evaluación con el RAVEN.

Se realizó un primer estudio, evaluando a 240 sujetos entre estudiantes de centros pre-universitarios, y trabajadores de universidades (UNMSM y la UNI) entre tutores y profesores. A continuación mostramos los resultados obtenidos. 
Tabla N. ${ }^{\circ}$ 1. Estadísticos generales de la aplicación del RAVEN

\begin{tabular}{cccc}
\hline & TOTAL PROMEDIO & Adolescentes & Adultos \\
\hline Media & 28.895 & 28.77 & 29.02 \\
Mediana & 28 & 28 & 28 \\
Moda & 28 & 28 & 28 \\
Máximo & 32.5 & 33 & 32 \\
Mínimo & 25.5 & 25 & 26 \\
D. T. & 4.25 & 4.3 & 4.2 \\
\hline
\end{tabular}

Estos son los resultados generales de la primera prueba aplicada $(\mathrm{N}=240$, media total alcanzada por los participantes fue de 29 , la mediana y moda coinciden en puntaje 28), en base a esos resultados se hacen las subdivisiones correspondientes para la división de la muestra y la aplicación correspondiente de la fase 2 .

Tabla N. ${ }^{\circ}$ 2. Media de los puntajes directos obtenidos según los grupos a considerar en la siguiente fase $(\mathrm{N}=120)$.

\begin{tabular}{lcccc}
\hline & \multicolumn{2}{c}{ Adolescentes } & \multicolumn{2}{c}{ Adultos } \\
\hline & $\begin{array}{c}\text { Para la aplicación } \\
\text { clásica }\end{array}$ & $\begin{array}{c}\text { Para la aplicación } \\
\text { informatizada }\end{array}$ & $\begin{array}{c}\text { Para la aplicación } \\
\text { clásica }\end{array}$ & $\begin{array}{c}\text { Para la aplicación } \\
\text { informatizada }\end{array}$ \\
Media & 28.88 & 29 & 29.01 & 28.92 \\
Mediana & 28 & 28 & 28 & 28 \\
Moda & 28 & 28 & 28 & 28 \\
Máximo & 31 & 31 & 31 & 31 \\
Mínimo & 27 & 27 & 27 & 27 \\
D. T. & 3.9 & 4.1 & 4.1 & 4 \\
\hline
\end{tabular}

Mediante el presente cuadro podemos observar que la distribución que se asigna para cada grupo según el tipo de modalidad de aplicación, en donde los adolescentes $(\mathrm{N}=60)$ y adultos $(\mathrm{N}=60)$ han sido seleccionados en base a aquellos que consiguieron puntajes similares en la aplicación del Raven para de este modo considerar homogeneidad entre los grupos. 
Tabla N. ${ }^{\circ}$ 3. Diferencia de Medias entre el grupo A (a quien se aplicó el test bajo la modalidad clásica) y el grupo B (a quien se aplicó el test bajo la modalidad informatizada).

\begin{tabular}{|c|c|c|c|c|c|c|c|}
\hline & \multicolumn{7}{|c|}{ Prueba T para la igualdad de medias } \\
\hline & \multirow[t]{2}{*}{$t$} & \multirow[t]{2}{*}{ gl } & \multirow[t]{2}{*}{$\begin{array}{c}\text { Sig. } \\
\text { (bilateral) }\end{array}$} & \multirow[t]{2}{*}{$\begin{array}{l}\text { Diferencia } \\
\text { de medias }\end{array}$} & \multirow[t]{2}{*}{ ET } & \multicolumn{2}{|c|}{$\begin{array}{l}\text { 95\% Intervalo de } \\
\text { confianza para la } \\
\text { diferencia }\end{array}$} \\
\hline & & & & & & Inferior & Superior \\
\hline $\begin{array}{l}\text { Puntajes } \\
\text { Raven para } \\
\text { los grupos } \\
\text { A y B }\end{array}$ & 1.46 & 80 & 0.25 & 3.09 & 1.46 & -0.56 & 4.04 \\
\hline
\end{tabular}

Como podemos apreciar en la Tabla N. ${ }^{\circ} 3$ no se encuentran diferencias significativas entre ambos grupos de evaluación. $(\mathrm{p}=0.25>\mathrm{p}=0.0)$

Tabla N. ${ }^{\circ}$ 4. Diferencia de medias de puntaje obtenido según los grupos de modalidad de aplicación en adolescentes.

\begin{tabular}{|c|c|c|c|c|c|c|c|}
\hline & \multicolumn{7}{|c|}{ Prueba T para la igualdad de medias } \\
\hline & \multirow[t]{2}{*}{$\mathrm{t}$} & \multirow[t]{2}{*}{ gl } & \multirow[t]{2}{*}{$\begin{array}{l}\text { Sig. } \\
\text { (bilateral) }\end{array}$} & \multirow[t]{2}{*}{$\begin{array}{l}\text { Diferencia } \\
\text { de medias }\end{array}$} & \multirow[t]{2}{*}{$\begin{array}{c}\text { Error } \\
\text { típ. de la } \\
\text { diferencia }\end{array}$} & \multicolumn{2}{|c|}{$\begin{array}{c}95 \% \text { Intervalo de } \\
\text { confianza para la } \\
\text { diferencia }\end{array}$} \\
\hline & & & & & & Inferior & Superior \\
\hline $\begin{array}{c}\text { Grupos de } \\
\text { aplicación } \\
\text { clásica e } \\
\text { informatizada } \\
\text { (RAVEN) }\end{array}$ & 1.16 & 80 & 0.25 & 1.67 & 1.43 & -1.19 & 4.52 \\
\hline
\end{tabular}

En esta tabla se puede observar que no existen diferencias significativas entre los grupos que se aplicó la prueba en la modalidad clásica y aquellos que desarrollaron el test en modalidad informatizada; con respecto a sus puntajes en la prueba del Raven, lo cual muestra mayor igualdad entre estos sujetos, la muestra es del grupo de adolescentes $(\mathrm{N}=60)$.

Tabla N. ${ }^{\circ} 5$. Diferencia de medias de puntaje obtenido según los grupos de modalidad de aplicación en adultos.

\begin{tabular}{|c|c|c|c|c|c|c|c|}
\hline & \multicolumn{7}{|c|}{ Prueba T para la igualdad de medias } \\
\hline & \multirow[t]{2}{*}{$t$} & \multirow[t]{2}{*}{ gl } & \multirow[t]{2}{*}{$\begin{array}{c}\text { Sig. } \\
\text { (bilateral) }\end{array}$} & \multirow[t]{2}{*}{$\begin{array}{l}\text { Diferencia } \\
\text { de medias }\end{array}$} & \multirow[t]{2}{*}{$\begin{array}{l}\text { Error típ. de } \\
\text { la diferencia }\end{array}$} & \multicolumn{2}{|c|}{$\begin{array}{c}95 \% \text { Intervalo de } \\
\text { confianza para la } \\
\text { diferencia }\end{array}$} \\
\hline & & & & & & Inferior & Superior \\
\hline $\begin{array}{l}\text { Grupos de } \\
\text { aplicación } \\
\text { clásica e } \\
\text { informatizada } \\
\text { (RAVEN) }\end{array}$ & 0.5 & 80 & 0.62 & 0.8 & 1.6 & -2.39 & 3.99 \\
\hline
\end{tabular}


En esta tabla se puede observar que no existen diferencias significativas entre los grupos que se aplicó la prueba en la modalidad clásica y aquellos que desarrollaron el test en modalidad informatizada; con respecto a sus puntajes en la prueba del Raven, lo cual muestra mayor igualdad entre estos sujetos, la muestra es del grupo de adultos $(\mathrm{N}=60)$.

Resultados de la evaluación con el TONI 2

Esta prueba se aplicó a 120 sujetos, dicho grupo fue seleccionado en base a la homogeneidad de los resultados de la primera prueba de inteligencia. Se obtuvieron los siguientes datos.

Tabla N. ${ }^{\circ}$ 6. Estadísticos generales de la aplicación del TONI 2.

\begin{tabular}{lccc}
\hline & TOTAL PROMEDIO & Adolescentes & Adultos \\
\hline Media & 44.965 & 44.78 & 45.15 \\
Mediana & 39 & 39 & 39 \\
Moda & 38.5 & 39 & 38 \\
Máximo & 51.5 & 52 & 51 \\
Mínimo & 35.5 & 34 & 37 \\
D. T. & 3.4 & 3.3 & 3.5 \\
\hline
\end{tabular}

Los puntajes obtenidos son considerablemente altos, lo cual refleja una similitud con los resultados obtenidos por el Raven (considerando que en ambas evaluaciones los sujetos sacaron puntuaciones altas, tener en cuenta que el puntaje máximo a lograr en el Raven es de 36 mientras que en el Toni es de 55).

Tabla N. ${ }^{\circ}$ 7. Diferencia de medias entre el grupo A (a quien se aplicó el test bajo la modalidad clásica) y el grupo B (a quien se aplicó el test bajo la modalidad informatizada).

\begin{tabular}{lccccc}
\hline & \multicolumn{5}{c}{ Prueba T para la igualdad de medias } \\
\cline { 2 - 6 } & $\mathrm{t}$ & $\mathrm{gl}$ & \multicolumn{1}{c}{ Sig. (bilateral) } & Diferencia de medias & $\mathrm{ET}$ \\
\hline Puntuaciones Toni 2 & 2.19 & 96 & 0.03 & 0.37 & 0.17 \\
\hline Grupos de evaluación & Aplicación informatizada & 39.36 & 3.21 & 0.48 \\
Toni 2 & Aplicación clásica & 38.03 & 4.72 & 0.71 \\
\hline
\end{tabular}

En el presente cuadro podemos observar una diferencia significativa entre los resultados (a un nivel de significancia de 0.05) entre ambas formas de aplicación, en donde se aprecia que la puntuación mediante la aplicación informatizada es ligeramente superior (en términos globales) que la aplicación clásica, considerando entonces que existe una diferencia significativa según la modalidad de aplicación $(\mathrm{p}=0.03<0=0.05)$. 
Tabla N ${ }^{\circ} 8$. Diferencia de medias de puntaje obtenido según los grupos de edad (adolescentes y adultos) en los dos tipos de aplicación.

\begin{tabular}{cccccc}
\hline & \multicolumn{5}{c}{ Prueba T para la igualdad de medias } \\
\cline { 2 - 6 } & $\mathrm{t}$ & $\mathrm{gl}$ & Sig. (bilateral) & Diferencia de medias & $\mathrm{ET}$ \\
\hline Grupo de adolescentes & 2.39 & 96 & 0.00 & 0.49 & 0.21 \\
\hline Grupo de adultos & 0.69 & 96 & 0.48 & 0.11 & 0.15 \\
\hline
\end{tabular}

En el presente cuadro se muestra si existe o no diferencia significativa en los resultados obtenidos, según la modalidad de aplicación, en los grupos de edades.

Se puede apreciar que si existe una diferencia significativa $(\mathrm{p}<0.00)$ en el grupo de los adolescentes, lo cual hace mención que si existen diferencias entre los adolescentes que desarrollaron su test en escrito a aquellos que lo desarrollaron en la computadora.

Por otro lado, con respecto al grupo de los adultos, no se detecta diferencia significativa entre ambos grupos según la modalidad de aplicación.

Tabla N. ${ }^{\circ}$ 9. Tabla de confiabilidad según el tipo de aplicación.

\begin{tabular}{lcc}
\hline & Alfa de Cronbach & N \\
\hline Aplicación informatizada & 0.811 & 60 \\
Aplicación clásica & 0.781 & 60 \\
\hline
\end{tabular}

En la tabla presentada se puede apreciar que, en las modalidades de aplicación, la aplicación informatizada tiene mayor puntaje que la clásica, lo cual se observa una diferencia que no es "grande" entre ambas puntuaciones, ya que redondeando a los decimales serían los mismos puntajes, de todas maneras es imprescindible tener estos datos en cuenta para futuras investigaciones que permitan profundizar este aspecto.

Tabla N. ${ }^{\circ} 10$ Tabla de validez de ítems total test según el tipo de aplicación.

\begin{tabular}{cccc}
\hline & $\mathrm{N}$ & $\begin{array}{c}\text { Correlación elemento-total } \\
\text { corregida }\end{array}$ & $\begin{array}{c}\text { Alfa de Cronbach si se elimina } \\
\text { el elemento }\end{array}$ \\
\hline $\begin{array}{c}\text { Promedio de los 55 ítems } \\
\text { (informatizado) }\end{array}$ & 60 & 0.66 & 0.781 \\
$\begin{array}{c}\text { Promedio de los 55 ítems } \\
\text { (clásico) }\end{array}$ & 60 & 0.46 & 0.811 \\
\hline
\end{tabular}

En esta tabla podemos identificar que los ítems de la prueba efectuada en modalidad informatizada difiere en puntajes con la modalidad clásica, esta diferencia no es "grande" pero de todas maneras es preciso considerar que sí se encuentran resultados diferentes. Propicio para ahondar el tema y poder hacer un estudio más detallado de este aspecto. 


\section{CONCLUSIONES}

Existen diferencias significativas entre los puntajes obtenidos por los sujetos según la modalidad de aplicación (la versión clásica con la versión informatizada de la prueba), esta diferencia no es muy remarcada; pero se puede decir que influye la modalidad de aplicación sobre los puntajes a obtener en el proceso de evaluación del test de inteligencia. Los mismos sujetos no muestran diferencia significativa en sus puntuaciones bajo la aplicación de la misma modalidad que se llevó a cabo en la primera evaluación con el Raven, por el contrario de los resultados obtenidos según las modalidades de aplicación. (Teniendo en cuenta que ambas pruebas utilizan ítems similares y la semejanza entre estos test es alta).

La diferencia en las puntuaciones, según la modalidad de aplicación, es más clara y evidente en el grupo de adolescentes que el de adultos, la diferencia de medias de estos grupos (modalidad de aplicación) es significativa a un nivel de significancia de 0.00 en el grupo de adolescentes, mas no es significativa en el grupo de adultos. Esto puede ser a causa de que dicha generación se encuentran mucho más familiarizados con la tecnología actual (sujeto a mayores investigaciones).

También se observan diferencias con respecto a los puntajes de confiabilidad y validez de ítems, estas diferencias no son remarcadas, no distan mucho los puntajes de un grupo con otro, de todas maneras es considerable que existan diferencia con respecto a estos valores dependiendo de la modalidad de aplicación del test. Esto invita a mayores investigaciones con más detenimiento en estos aspectos.

\section{REFERENCIAS BIBLIOGRÁFICAS}

Alarcón, R. (1991). Métodos y diseños de investigación del comportamiento. Lima: Fondo editorial.

Aiken, L. (1995). Tests psicológicos de evaluación. México: Prentice-Hall.

Anstey, E. (1976). Los tests psicológicos. Madrid: Marova.

Brown, G. F. (1980). Principios de la medición en psicología y educación.

Calero, M. (1995). Modificación de la inteligencia. España: Ed. Ediciones Pirámide.

Eysenck, H. (1983). Estructura y medición de la inteligencia. Barcelona. Ed. Herder.

Gardner, H. (1998). Inteligencias múltiples. Madrid. Ed. Grafiques 92, S.A.

Gardner, (1999). Estructura de la mente.Colombia: Fondo de Cultura Económica.

Kaufman, A. (1997). Nuevas alternativas para la interpretación del Wisc III.

Yuste, C. y Otros. (1995). Programas para la estimulación de las habilidades de inteligencia. España: Ed. CEPE.

Lewis, A. (1996). Test psicológicos y evaluación. México: Ed. Prentice Hall.

Nieto, J. (1998). Como enseñar a pensar. España: Ed. Escuela Española. 
Simmons, S. ySimmons, J. (1998). Cómo medir la inteligencia emocional. Madrid. Editorial EDAF.

Sternberg, R. (1997). La inteligencia exitosa. Barcelona. Paidós.

Sattler, J. (1988). Evaluación de la inteligencia infantil y habilidades especiales. México: Ed. Manual Moderno. Thorndike, R. (1996).

Tyler, L. (1972). Pruebas y medición en psicología. Madrid, España: Prentice-Hall International.

Yuste, C. y Otros. (1995). Programas para la estimulación de las habilidades de inteligencia. España: Ed. CEPE. 